\title{
BUCLM
}

Ediciones de la Universidad

Técnicas de investigación cualitativa en los ámbitos sanitario y sociosanitario

Edición preparada por: Jesús Manuel Tejero González 



\section{TÉCNICAS DE INVESTIGACIÓN CUALITATIVA EN LOS ÁMBITOS SANITARIO Y SOCIOSANITARIO}





\section{TÉCNICAS DE INVESTIGACIÓN CUALITATIVA EN LOS ÁMBITOS SANITARIO Y SOCIOSANITARIO}

Edición preparada por:

Jesús Manuel Tejero González

(1) $\frac{\text { Ediciones de la Universidad }}{\text { de Castilla-La Mancha }}$

Cuenca, 2021 
TÉCNICAS de investigación cualitativa en los ámbitos sanitario y sociosanitario / edición preparada por Jesús Manuel Tejero González.- Cuenca : Ediciones de la Universidad de Castilla-La Mancha, 2021

180 p. ; 24 cm.- (Estudios ; 171)

ISBN 978-84-9044-423-8

1. Investigación médica 2. Medicina - Relación médico/paciente 3. Investigación y teoría de la enfermería I. Tejero González, Jesús Manuel, ed. lit. II. Universidad de Castilla-La Mancha, ed. III. Título IV. Serie

614.253 .8

614.253 .5

MBGR - MBDP - MQCB

Cualquier forma de reproducción, distribución, comunicación pública o transformación solo puede ser realizada con la autorización de EDICIONES DE LA UNIVERSIDAD DE CASTILLA-LA MANCHA salvo excepción prevista por la ley.

Diríjase a CEDRO (Centro Español de Derechos Reprográficos - www.cedro.org), si necesita fotocopiar o escanear algún fragmento de esta obra.

(C) de los textos e imágenes: sus autores.

(C) de la edición: Universidad de Castilla-La Mancha.

Edita: Ediciones de la Universidad de Castilla-La Mancha.

Colección ESTUDIOS n. ${ }^{\circ} 171$

ISSN: 2255-2618 / ISSN-L: 2255-2618

I.S.B.N.: 978-84-9044-423-8 (Edición impresa)

I.S.B.N.: 978-84-9044-424-5 (Edición electrónica)

D.O.I.: http://doi.org/10.18239/estudios_2021.171.00

D.L.: CU 13-2021

Diseño de la colección y de la cubierta:

C.I.D.I. (Universidad de Castilla-La Mancha)

11e Esta editorial es miembro de la UNE, lo que garantiza la difusión y

comercialización de sus publicaciones a nivel nacional e internacional.

Composición: Compobell, S.L.

Impresión: MG Color

Esta obra se encuentra bajo una licencia internacional Creative Commons BY-NC-ND 4.0.

Cualquier forma de reproducción, distribución, comunicación pública o transformación de esta obra no incluida en la licencia Creative Commons BY-NC-ND 4.0 solo puede ser realizada con la autorización expresa de los titulares, salvo excepción prevista por la ley. Puede usted acceder al texto completo de la licencia en este enlace: https://creativecommons.org/licenses/by-nc-nd/4.0/deed.es

Hecho en España (U.E.) - Made in Spain (E.U.) 


\section{ÍNDICE}

Capítulo 1. Introducción al método científico. Paradigmas empíricos e inter-

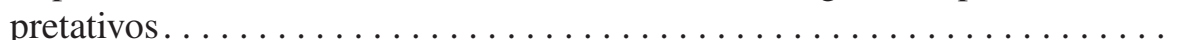

J. Manuel Tejero González

Capítulo 2. La investigación-acción como método de evaluación participativa. M. Pilar Delgado Hito

Capítulo 3: El método Delphi .................................

Mercedes Guilabert Mora

Capítulo 4. Entrevistas estructuradas, semi-estructuradas y libres. Análisis de

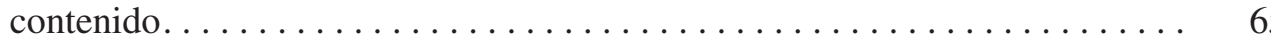

Raquel Lázaro Gutiérrez

Capítulo 5. Observación participante. Historias de vida y entrevista en pro-

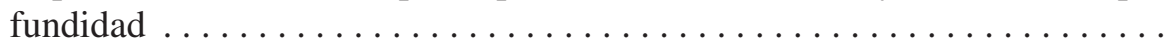
Rosa Castro Ramos

Capítulo 6. Grupos de discusión y grupos focales $M^{a}$ Virtudes Pérez Jover

Capítulo 7. Encuestas de opinión Isabel María Navarro Soler

Capítulo 8. Calidad percibida y satisfacción: Cómo conocer y medir la experiencia del paciente en una organización sanitaria $\ldots \ldots \ldots \ldots \ldots \ldots \ldots$ Encarnación Añó Consuegra

Capítulo 9. Identificación de evidencia científica y sus repercusiones en la

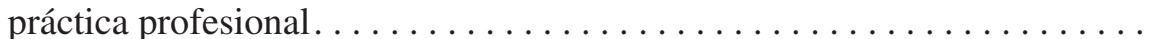

Francisco Alberto García-Sánchez 



\title{
CAPÍTULO 8
}

\section{CALIDAD PERCIBIDA Y SATISFACCIÓN: CÓMO CONOCER Y MEDIR LA EXPERIENCIA DEL PACIENTE EN UNA ORGANIZACIÓN SANITARIA}

\author{
ENCARNACIÓN AÑÓ CONSUEGRA
}

SESCAM

\section{INTRODUCCIÓN}

La década pasada ha traído un creciente interés en la comprensión de cómo las personas experimentan los servicios de salud. En una organización orientada al paciente y a la mejora de la atención que presta, se hace necesario el uso de herramientas que nos permitan recopilar información sobre la experiencia personal del paciente respecto a su proceso asistencial, aproximarnos a sus expectativas, y detectar aquellos aspectos que sugieren mejorables, para poder así implementar mejoras en la gestión. Conocer, analizar y mejorar la experiencia del paciente en un servicio de salud, debe ser objetivo clave en las organizaciones sanitarias.

En los sistemas sanitarios, ésta es una de las líneas estratégicas básicas en las que se trabaja en los Servicios de Atención al Usuario; desempeñan el papel de "sensor de opinión" en la organización, de cara a proyectar la voz de los pacientes en la organización y potenciar la puesta en marcha de acciones que logren mejorar su satisfacción. En otras palabras, detectar aquellos aspectos que los grupos de interés sugieren mejorables, para poder así implementar mejoras en la gestión centradas en el paciente. 
Existen formas diversas de conocer y "medir" la experiencia del paciente, y que buscan asimismo determinar perfiles de usuarios satisfechos/insatisfechos describiendo qué elementos/dimensiones están condicionando la satisfacción y en qué medida. En este tema, nos proponemos adentrar en algunas de estas herramientas metodológicas de corte cuantitativo y cualitativo ya vistas en temas anteriores, aplicándolas al campo de la calidad percibida y satisfacción del usuario en un servicio público de salud.

Como veremos, son muchas las técnicas que se pueden aplicar para conocer y analizar la experiencia del paciente, pero es posible establecer cuál de ellas es más efectiva o mejor, ya que cada una sugiere unos pros y contras que también intentaremos describir.

\section{CALIDAD PERCIBIDA Y GESTIÓN ORIENTADA AL PACIENTE}

La Organización Mundial de la Salud (OMS) considera que el concepto de Calidad Asistencial en los Servicios de Salud debe incluir aspectos como la excelencia profesional, eficiencia de los recursos, seguridad del paciente, resultados en salud, así como el grado de satisfacción por parte del paciente. Efectivamente, en el ámbito de la prestación de los servicios sanitarios, la calidad asistencial puede definirse a partir de dos dimensiones básicas: Calidad científico-técnica y Calidad percibida.

Cuando hablamos de calidad científico-técnica, nos referimos a aquellas características técnicas o funcionales del servicio prestado. Se evalúan aspectos tales como la eficacia, efectividad, adecuación, competencia técnica y seguridad, siendo medible y cuantificable a partir del cumplimiento de unos estándares definidos, y por tanto, comparable desde un punto de vista técnico. Como vemos, la objetividad es una de las características de la calidad científico-técnica.

Pero unido a estas dimensiones, existen otros aspectos que el paciente/familiar valora también respecto a la atención que recibe, y que van conformando una percepción subjetiva y personal de todo su proceso asistencial, La calidad percibida procede del juicio del usuario, evaluando de forma amplia la satisfacción respecto a otros elementos más allá de los científico-técnicos tales como la información, la accesibilidad al sistema sanitario, el trato, el confort, la continuidad de cuidados o la capacidad de respuesta. En este sentido, se entiende como la diferencia entre lo que el usuario espera/desea (sus expectativas) y lo que percibe u obtiene, o como "la apreciación que hace el paciente de la adecuación, resultados y beneficios del

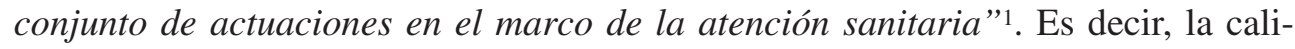

1 Mira JJ. y Aranaz, J. La satisfacción del paciente como una medida del resultado de la atención sanitaria. Med Clin 2000; 114 (Supl 3):26-33. 
dad percibida podemos resumirla en la evaluación (subjetiva) que los pacientes/ familiares realizan de la totalidad de su proceso asistencial.

Por último, para cerrar la introducción de este concepto de calidad asistencial, cabe mencionar que estas dos dimensiones de la calidad no son sustitutivos, y únicamente podremos trabajar en mejorar la Calidad Percibida, cuando se parta de una calidad objetiva alta en la organización, pues trabajar en estos aspectos de la calidad percibida, cuando se está prestando un servicio de baja calidad técnico-asistencial, obviamente irrita al usuario.

Nos encontramos pues, con que el concepto de Calidad Percibida, tiene mucho que ver con la gestión orientada al paciente, pues uno de los requisitos fundamentales para el éxito en cualquier organización, y también en la sanitaria, es identificar a los grupos de interés, conocer sus necesidades/expectativas, y orientar la toma de decisiones y actuaciones en gestión hacia éstas.

Aunque tradicionalmente sabemos que desde las organizaciones sanitarias no se ha considerado importante la participación de paciente en este sentido, hoy es plenamente aceptado que las organizaciones deben orientarse a sus grupos de interés prioritarios, y bajo este precepto la utilización de herramientas y fuentes de información que nos permitan conocer la satisfacción del paciente respecto a la asistencia recibida y acercarnos a su experiencia como pacientes/familiares, debe constituir uno de los motores imprescindibles para guiar la gestión de los servicios de salud y mejorar así la atención que se presta en función de los requerimientos del usuario.

En este sentido, cabe destacar la existencia de estudios que respaldan la importancia de medir la satisfacción del paciente como uno de los pilares básicos de la calidad en temas de salud, estableciendo incluso relaciones entre ésta y los resultados clínicos ${ }^{2}$; este es el caso de una revisión sistemática de 55 estudios en atención primaria y especializada que se realizó en el Reino Unido, evidenciando vínculos entre la experiencia del paciente, la seguridad del paciente y la efectividad clínica. Todo ello otorgaría soporte al argumento de que las dimensiones de la calidad deben ser tomadas como un conjunto y no de forma aislada ${ }^{3}$.

En los servicios de salud, tal y como avanzábamos en la introducción, ésta es una de las líneas estratégicas básicas de trabajo en los Servicios de Atención al Usuario; desempeñan el papel de "sensor de opinión" en la organización, de cara a proyectar la voz de los pacientes en la organización y potenciar la puesta en marcha de acciones que logren mejorar la satisfacción de los/as usuarios/as.

2 Riskind P, Fossey L, Brill K (2011). Why measure patient satisfaction? J Med Pract Manage; 26.

3 Doyle C, Lennox L, Bell D (2013). A systematic review of evidence on the links between patient experience and clinical safety and effectiveness. BMJ Open; 3. 


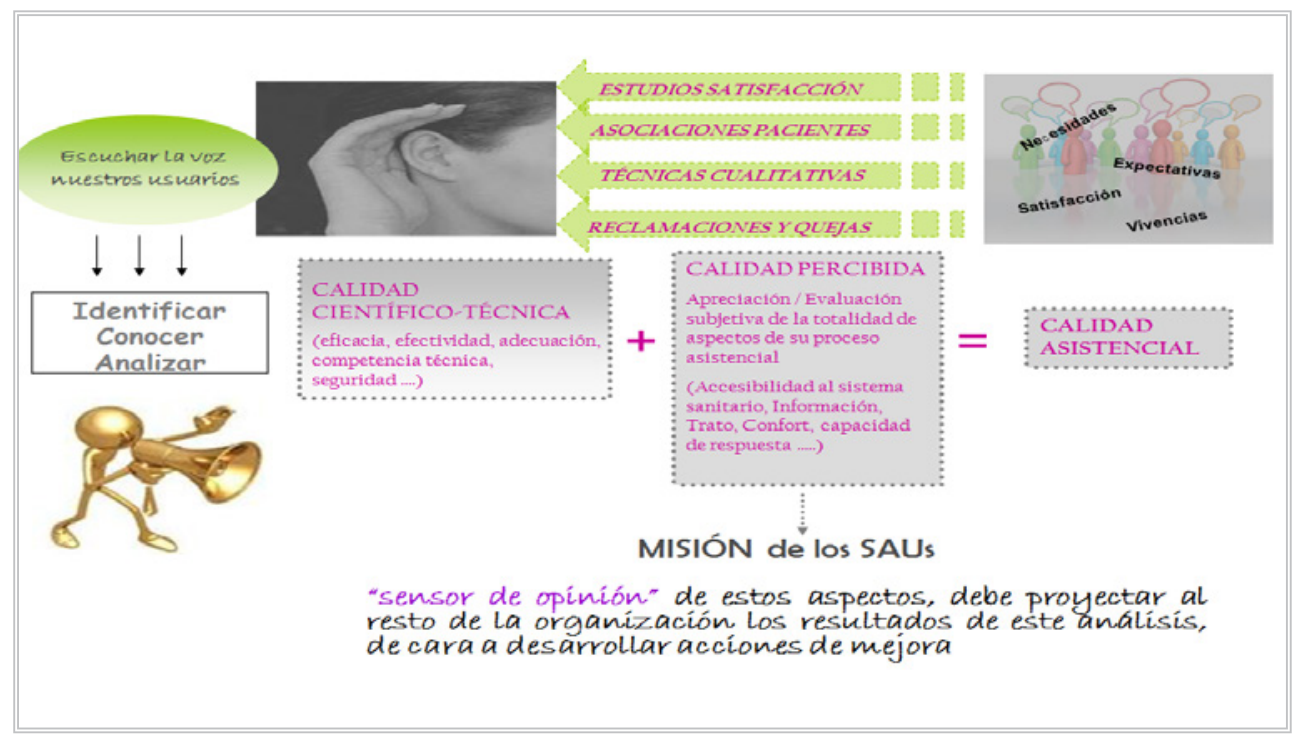

Figura I: Escuchando la voz de los usuarios en la organización.

En esta línea, otra de las estructuras básicas en los Servicios de Salud como marco para el trabajo de estos aspectos, son los Comités de Calidad Percibida. El Comité de Calidad Percibida, es una estructura funcional multidisciplinar existente en algunos Centros Sanitarios, cuyo objetivo general es conocer y mejorar la calidad percibida por los pacientes y familiares; mencionar brevemente algunas de las líneas de trabajo clave que se desarrollan desde estos comités:

- Analizar las opiniones de los pacientes/familiares e identificación de áreas de mejora, a través del desarrollo de canales de información y técnicas que permitan conocer y medir la percepción de los pacientes (grupos focales, entrevistas, encuestas, asociaciones de pacientes.....)

- Priorizar, definir y proponer líneas de trabajo en calidad percibida, de acuerdo con las áreas de mejora identificadas y la viabilidad de las mismas.

- Dar a conocer y sensibilizar a los profesionales sobre la importancia de la opinión del usuario en la mejora de la calidad, promoviendo la cultura de la Calidad en la organización.

- Disponer de un espacio institucional de recogida, debate y análisis de problemas relacionados con la calidad percibida por los usuarios y acompañantes,

- Colaborar con otros grupos de trabajo, comisiones y equipos directivos, orientando la gestión a las expectativas y necesidades de los ciudadanos. 


\section{3. ¿QUÉ ENFOQUE METODOLÓGICO UTILIZAR, CUANDO TRATA- MOS DE MEDIR Y ANALIZAR EXPERIENCIAS Y SATISFACCIÓN?}

La medición de la experiencia del paciente y la puesta en marcha de métodos y canales para escuchar su voz, "constituye una revolución silenciosa que paulatinamente va introduciendo un cambio en la cultura de las organizaciones"4. Pero, medir la experiencia del paciente respecto a su vivencia asistencial no es objeto de estudio fácil; por ejemplo en ocasiones, y no pocas, el/la paciente puede reportar altos niveles de satisfacción, y al mismo tiempo describir experiencias mucho menos óptimas ${ }^{5}$ ¿ ¿Cómo escuchar desde una Organización sanitaria a los/as pacientes?, ¿Qué técnicas de investigación utilizar? ¿Existe evidencia sobre el mejor uso de éstas en la obtención de resultados?

Tal y como hemos visto en los capítulos anteriores, podemos resumir que el objetivo de la investigación cualitativa, es entender, profundizar e interpretar la realidad, centrándose por tanto en actitudes, creencias, opiniones, motivaciones; por otra parte, la investigación cuantitativa, podemos decir que su objetivo principal es medir centrándose más en responder a cuestiones como el donde, el cuando el cómo y el quien....

Hemos visto, que en líneas generales existe cierto consenso en las ciencias sociales en cuanto al uso de técnicas cuantitativas y cualitativas de modo complementario, y no como enfoques metodológicos opuestos entre sí. En la medición de la opinión/satisfacción del paciente, si más cabe esta concepción: dado el alto grado de complejidad que supone la propia vivencia de un proceso asistencial, la combinación de ambos métodos y diversas fuentes de información nos permiten un mayor acercamiento a esa realidad sentida por el paciente. A groso modo podemos decir, que si las técnicas cuantitativas en datos numéricos logran aproximarnos de forma "superficial" identificando incluso un sentir "general", el enfoque cualitativo, nos permite profundizar en esas vivencias, experiencias... indagando a través de una retroalimentación mayormente descriptiva.

4 Sociedad española de Atención al Usuario de la Sanidad (2010). Claves para la Gestión de la Atención al Usuario en los Servicios de Salud.

5 Byrne K, Sims-Gould J, Frazee K, Martin-Matthews A (2011). "I'm satisfied ... but”: clients' and families'contingent responses about home care. Home Health Care Serv Q; 30. 


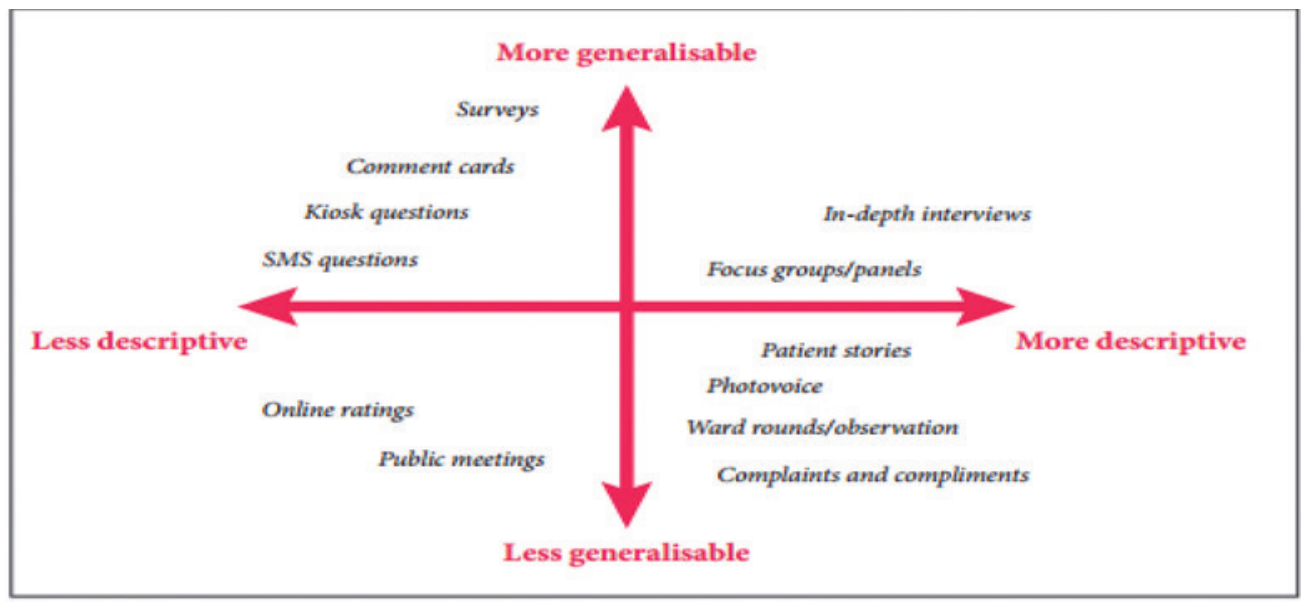

Figura II. Ejemplos de métodos utilizados para medir la experiencia del paciente ${ }^{6}$.

Por tanto, un buen uso en la combinación de estos enfoques y técnicas, supone una enriquecedora complementación en la búsqueda de resultados de satisfacción. Una breve encuesta puede ayudarnos a disponer de una visión sobre tendencias generales que se pueden cuantificar y comparar en el tiempo; si a esto le sumamos algunas entrevistas o un grupo focal orientado a indagar en aquellos aspectos que la encuesta ya ha identificado, obtendremos una mayor comprensión del por qué de esos datos y del sentir de esa población atendida respecto a esos aspectos.

¿Pero cuando utilizar unas técnicas u otras? En principio, estos enfoques metodológicos como hemos ido viendo, pueden discernirse de acuerdo a la profundidad de información que proporcionan y al grado en que recogen información (generalizables a una población más amplia o no). En la selección de un enfoque apropiado de medición, puede ser necesario sopesar la importancia de la profundidad frente a la generalización, o combinar los enfoques para obtener una mezcla de ambos ${ }^{7}$.

Una de las cuestiones importantes que nos ayudará a la hora de establecer una estrategia de investigación para conocer la opinión del paciente, es qué aspectos de la experiencia queremos indagar...qué queremos medir.

Cómo hemos visto, la experiencia vivida, la satisfacción/insatisfacción percibida por el paciente es algo complejo y multidimensional; en la figura III, se muestran algunos de los aspectos que inciden en la percepeción del paciente respecto al servicio sanitario prestado:

6 De Silva, D. (2013) Measuring patient experience. Evidence Scan. The Health Foundation; 18.

7 De Silva, D. (2013) Measuring patient experience. Evidence Scan. The Health Foundation; N ${ }^{\circ}$ 18. 


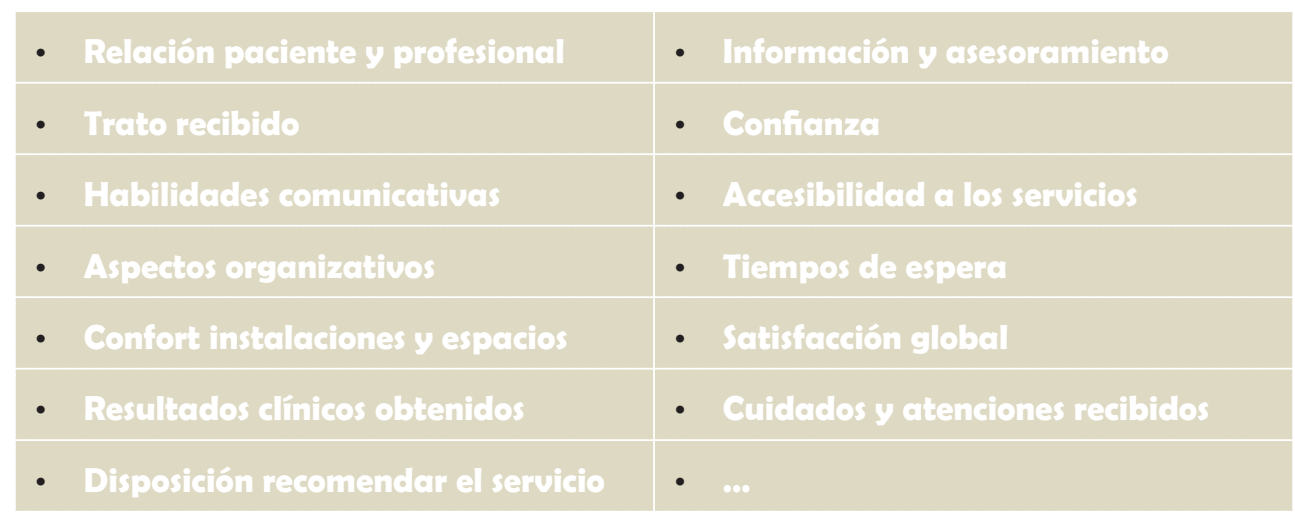

Figura III. Aspectos que conforman la calidad percibida por parte del paciente.

En este sentido, conocer estos aspectos y decidir cuál de ellos nos interesa abordar, nos facilitará también la elección de la herramienta a utilizar, pero verdaderamente no se puede confirmar que en este ámbito haya una mejor o más efectiva, teniendo todas sus pros y contras, por lo que deberemos también elegirlas en función del contexto, perfil de pacientes, recursos humanos y materiales, tiempos etc...

Veamos algunas de estas técnicas.

\section{4. ¿CÓMO ESCUCHAR LA VOZ DE NUESTROS/AS USUARIOS/AS?: FUENTES, TÉCNICAS Y USOS}

En esta unidad, nos proponemos reflejar la aplicabilidad de algunas de estas técnicas y canales de información que podemos utilizar en el ámbito de la calidad percibida, principalmente para conocer la opinión de los/as usuarios/as respecto a la asistencia y servicios prestados. Al mismo tiempo, intentaremos mostrar a modo de ejemplo, la experiencia en cuanto a la aplicación de algunas de estas herramientas en el Servicio de salud de Castilla la Mancha (SESCAM), y concretamente en la Gerencia de Atención Integrada de Ciudad Real.

Cada una de las herramientas, como decíamos muestra una serie de fortalezas y debilidades; intentaremos también desgranarlos a partir del análisis que realiza en el año 2013 The Health Foundation en Reino Unido ${ }^{8}$,a través de una revisión bibliográfica de 328 estudios empíricos de cada una de las herramientas, mostrando sus limitaciones y potencialidades.

8 De Silva, D. (2013) Measuring patient experience. Evidence Scan. The Health Foundation; N ${ }^{\circ}$ 18. 


\subsection{ANÁLISIS DE QUEJAS Y RECLAMACIONES formuladas por los usuarios/ as del Servicio de Salud.}

El análisis de las reclamaciones formuladas por los pacientes y usuarios en un centro sanitario, podemos entenderla como un método indirecto ya que está basado en recoger datos de una fuente ya establecida en un servicio de salud, como canal de información entre el paciente y la organización.

La explotación de estos datos, se hace impensable obviarlo, pues brindan la oportunidad de detectar problemáticas y conocer la opinión de los usuarios, y en este sentido, actualmente supone en los Servicios de Salud, una de las fuentes más instituidas para detectar posibles insuficiencias, si bien, todavía en muchos casos se atienden de forma superficial y a "disgusto" sin aprovechar su potencial transformador, al identificar oportunidades y áreas de mejora.

Entendemos la reclamación como la expresión de la opinión no satisfactoria de un usuario/a en relación con un acto o proceso asistencial o administrativo, en relación con su sistema sanitario. Es importante conocer, que la reclamación tiene su razón de ser como derecho de todo usuario/a de un Servicio de Salud, como forma de participación legalmente establecida y como una oportunidad de mejora, y por tanto, estrategia de la organización. Detengámonos en mostrar la doble dimensión que alberga:

a. Como derecho del paciente, dentro de un amplio marco normativo que lo avala. (Ver figura IV). En la Orden 20-02-03 sobre Quejas y Reclamaciones en el Servicio de Salud de Castilla la Mancha, así es como se define: "Se entiende por queja la simple comunicación de los usuarios de su insatisfacción por el funcionamiento de los servicios sanitarios, prestaciones recibidas, cuidado y atenciones. Puede tratarse de requerimientos de naturaleza sanitario-asistencial o no asistencial. Cuando dicha comunicación se acompaña de una demanda concreta sobre cualquiera de estos aspectos, se considera reclamación"

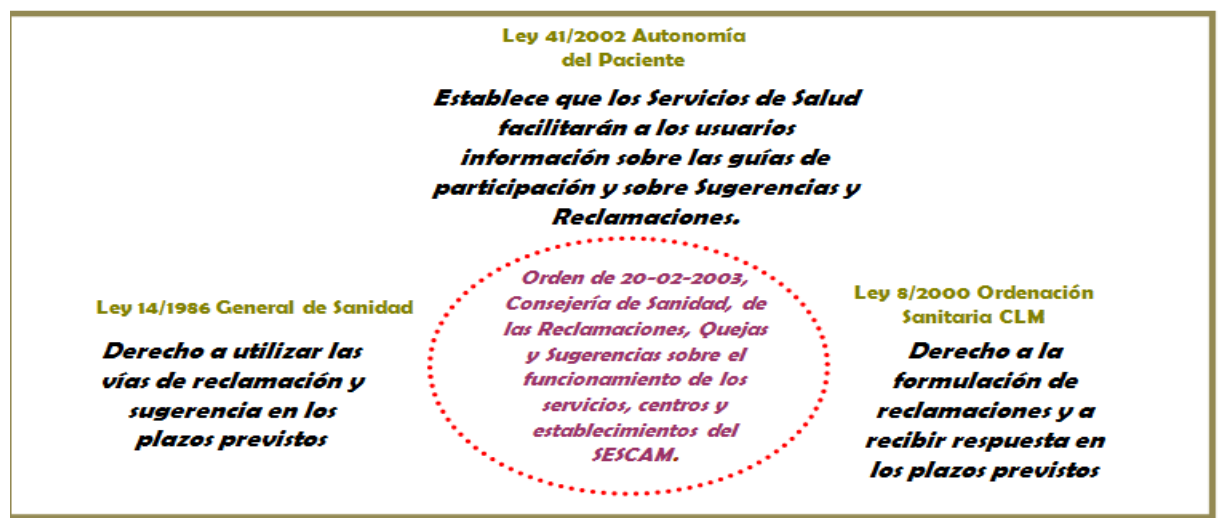

Figura IV. Marco Normativo de la Gestión de Reclamaciones. 
b. Como fuente de detección de áreas de mejora. La reclamación es también una oportunidad de mejora, la información que se obtiene de las reclamaciones dice mucho de la organización y es muy útil para identificar puntos críticos de mejora "La recopilación y análisis sistemático de las reclamaciones, debe utilizarse para reunir información sobre la calidad de la asistencia sanitaria, y como indicación de las áreas y aspectos que necesitan mejorarse" Recomendación $\mathrm{n}^{\circ} \mathrm{R} 5$ (2000) del Consejo de Europa.

En el Servicio de Salud de Castilla la Mancha (SESCAM), el análisis estadístico se realiza tomando como fuente de datos el registro de las reclamaciones que cada centro Sanitario de la región realiza en la aplicación Sistema de Gestión de Reclamaciones y Sugerencias (SGRS). A nivel cuantitativo se explotan los datos por número total de reclamaciones por centro sanitario y/o servicio, y codificado por categorías/motivo de reclamación (falta de información, trato recibido, lista de espera, disconformidad de la asistencia, demoras, limpieza etc...).

Concretamente, desde la experiencia de la Gerencia de Atención Integrada de Ciudad Real, se incorpora en esta explotación una nueva variable, la actividad asistencial de cada servicio, estableciendo Tasa de Incidencia de Reclamaciones, la denominada TIR (número de reclamaciones por cada 10.000 actos asistenciales). Conjugar reclamaciones y asistencia, en lugar de disponer únicamente de los datos de reclamaciones en bruto, nos permite disponer de una mayor precisión en el análisis de las tendencias, monitorizar un indicador numérico de calidad percibida y avanzar en la disposición de herramientas que nos permitan un benchmarking en el ámbito de las reclamaciones con otros centros sanitarios.

A partir de la explotación de estos datos por parte de los Servicios de Información y Atención al usuario, se debe generar y potenciar la transmisión de estos resultados a los puestos directivos y al resto de profesionales de la organización de cara a la puesta en marcha de acciones de mejora en los aspectos peor valorados. Cada servicio así, dispondrá por una parte de las tendencias anuales tanto de la TIR, como de cada una de las categorías y motivos de reclamación. La siguiente figura, muestra un ejemplo de los informes que se trasladan a los distintos Centros de Salud y Servicios, que muestra el evolutivo de la tasa y el número total de reclamaciones.

9 Ministerio de Sanidad y Consumo (2008). Análisis y benchmarking del tratamiento de las reclamaciones y sugerencias en el Sistema Nacional de Salud. 


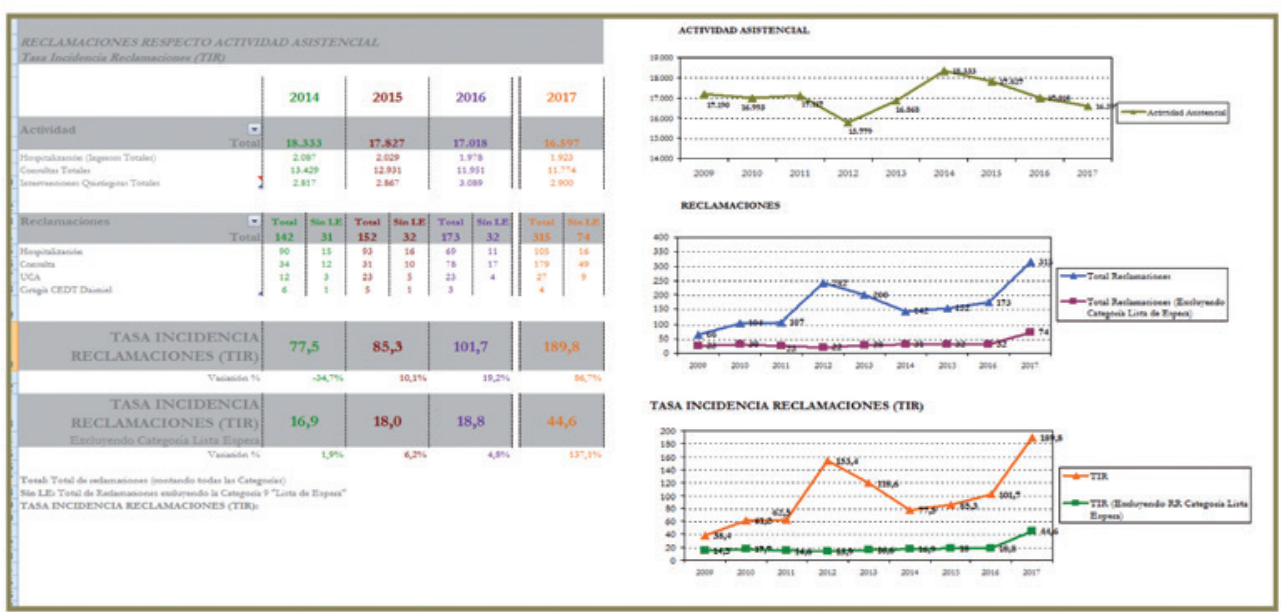

Figura V. Informes de reclamaciones por Servicios.

Para finalizar con esta fuente de información, mencionar algunas de las fortalezas y debilidades del uso de las reclamaciones como fuente de información para conocer la opinión de los pacientes. Por una parte, su análisis cuantitativo podemos decir que proporciona un "mapa general" del centro sanitario en cuanto a aquellos aspectos en que las personas sienten como verdaderamente preocupantes y por tanto mejorables, si bien hay que pensar un importante sesgo que es su tendencia hacia los aspectos más graves de la atención recibida, es decir, tienden a recoger las experiencias extremas y vivencias negativas, no se puede interpretar por tanto, como una opinión generalizable y se hace muy necesario complementarlo con otros métodos directos de indagación diseñados a tal fin, como veremos a continuación. 
Figura VI. Fortalezas y Limitaciones de medir la experiencia del paciente a través de las reclamaciones.

\begin{tabular}{|c|c|}
\hline Fortalezas & Limitaciones \\
\hline $\begin{array}{l}\text { Puede proporcionar información detallada } \\
\text { sobre aspectos que necesitan mejorar. } \\
\text { Permite identificar cuestiones que las } \\
\text { personas sienten verdadera preocupación, } \\
\text { al ser de reporte espontáneo. } \\
\text { Beneficiarse de una fuente de información } \\
\text { del paciente ya establecida en la } \\
\text { organización. } \\
\text { Traducir la visión de la reclamación como } \\
\text { una oportunidad de mejora, además de } \\
\text { derecho del paciente. }\end{array}$ & $\begin{array}{l}\text { Al tratarse de un sistema formal, puede } \\
\text { darse una tendencia a recoger experiencias } \\
\text { extremas. } \\
\text { Riesgo de enfocar el análisis en } \\
\text { experiencias muy individuales, en lugar de } \\
\text { aspectos más amplios de atención o } \\
\text { servicios. } \\
\text { No recoge los aspectos positivos y puntos } \\
\text { fuertes del servicio que se presta. }\end{array}$ \\
\hline
\end{tabular}

Fuente: Elaboración propia a partir de De Silva, D. (2013) Measuring patient experience. Evidence Scan. The Health Foundation; $18^{10}$

\subsection{Asociaciones de pacientes y grupos de autoayuda}

Al igual que las reclamaciones, el canal de comunicación que se establece con las Asociaciones de pacientes, contituye otro de los métodos (no directos) de aproximación al conocimiento de la opinión de los pacientes. Las Asociaciones de Pacientes y familiares (ASPF) constituyen un grupo de interés clave para el sistema sanitario, y en este sentido las organizaciones sanitarias destinan cada vez más recursos tanto materiales como capital humano al despliegue de proyectos de colaboración con los movimientos asociativos.

En esta línea, la implicación y participación de los movimientos asociativos en los sistemas sanitarios, son fundamentales en dos vertientes:

1. Como una de las fuentes principales de información en cuanto a detección de áreas de mejora para la organización (calidad percibida).

2. En el papel que desempeñan como soporte al paciente ofreciéndole unos servicios/ recursos determinados, que complementan en gran medida la atención sanitaria pública.

10 De Silva, D. (2013) Measuring patient experience. Evidence Scan. The Health Foundation; 18. 
Establecer un canal de comunicación con las Asociaciones de pacientes y familiares, supone disponer de una importante fuente de información de cara a conocer las necesidades y opiniones del paciente respecto a la atención que reciben. Por ello, se hace necesario potenciar el diálogo con las Asociaciones con la finalidad de detectar aspectos y propuestas de mejora en cuanto a la organización sanitaria, y trabajar conjuntamente en el desarrollo de actuaciones en beneficio de los/as pacientes.

Concretamente el Hospital General Universitario Ciudad Real en el año 2012, en el marco de la Memoria de sostenibilidad 2008-2011 ${ }^{11}$, potencia la apertura de un canal de comunicación con las asociaciones de pacientes con los propósitos anteriormente descritos. Para ello, en primer lugar se elabora un directorio de asociaciones del área de salud, informándoles sobre la iniciativa e invitándoles a participar. Se establece un primer contacto y reuniones de encuentro con informantes clave de la principales asociaciones utilizando la técnica cualitativa de la entrevista. Se diseña una entrevista semiestructurada, estableciendo un guión que incorpora los temas que queremos explorar, proponiéndonos recopilar información en tres líneas básicas, y realizando posteriormente un análisis descriptivo de las respuestas:

- Conocer el perfil de la asociación.

- Conocer las colaboraciones existentes hasta el momento entre la asociación y los servicios del hospital.

- Identificar áreas de mejora que detectan cada una de las asociaciones y recoger sus propuestas de colaboración futuras.

Para finalizar este apartado resaltar que hay un largo camino a recorrer en la línea de la participación ciudadana en temas de salud. Aunque las organizaciones de pacientes existen desde hace muchas décadas, en la actualidad, y en la mayor parte de las sociedades desarrolladas, se asiste a un período de auge de estos movimientos asociativos. Al mismo tiempo, este interés que venimos remarcando en conocer las preferencias de los pacientes ha dado lugar en algunos países, a tomar como uno de los ejes centrales en sus políticas la participación de las asociaciones en las decisiones que afectan al funcionamiento de los servicios sanitarios. Pero la realidad en la gran mayoría de países, es que el fomento de la participación de las asociaciones, no es consistente en las políticas sanitarias. De hecho, hay estudios que muestran esta "necesidad de un compromiso creíble de las autoridades sanitarias en la faci-

11 Mas información en http://www.hgucr.es/wp-content/hgucr/flash/hgucr.htm. 
litación de mecanismos y espacios de decisión y elección, así como de evaluación efectiva del funcionamiento de dichos mecanismos". ${ }^{12}$

\subsection{Encuestas de satisfacción}

Pasamos ya en este apartado, a desarrollar una de las técnicas ya directas de investifación: la encuesta de satisfacción.

La encuesta de satisfacción es tradicionalmente una de las herramientas más comúnmente utilizadas para la realización de estudios de opinión del consumidor/a de servicios, y en nuestro caso del paciente. Se considera uno de los medios más económicos y eficientes en la obtención de información por parte de los usuarios/as. El objetivo principal de la encuesta es medir la opinión del paciente sobre la atención recibida; es la voz del usuario en cifras.

Las encuestas de satisfacción en el caso del Servicio de Salud de Castilla La Mancha, son realizadas principalmente a nivel regional con periodicidad anual. Se toma como población de referencia usuarios atendidos por el SESCAM, realizando una distribución de la muestra (estratificada) por usuarios de Atención Primaria, Atención Especializada, Consultas Externas y Urgencias Hospitalarias.

Las encuestas de satisfacción nos permiten ${ }^{13}$ :

- Obtener información cuantitativa sobre percepciones y satisfacción global con el servicio recibido por parte de los usuarios y valoraciones sobre calidad percibida.

- Detectar problemas y áreas de mejora en la calidad de la atención, obteniendo valoraciones en cuanto a aspectos tales como: atención recibida de los profesionales, medios e infraestructuras disponibles, accesibilidad, condiciones de prestación de los servicios, organización y funcionamiento de éstos, seguridad y confianza que le merecen, trato personal, amabilidad y valoración de los tiempos hasta recibir la atención.

- Realizar análisis de tendencias interanuales de las principales variables, construyendo índices sintéticos de satisfacción que facilitan un seguimiento longitudinal en el futuro.

12 García-Sempere A., Artells J.J. Organización, funcionamiento y expectativas de las organizaciones representativas de pacientes: enuesta a informadores clave. Fundación Salud, Innovación y Sociedad. Barcelona. España.

13 SESCAM Servicio de Salud de Castilla-La Mancha. Área de Seguridad del Paciente y Calidad Percibida (2008) Estrategia de Confortabilidad en el SESCAM. 
La aplicación de esta técnica como vemos, aún no alcanzando profundidad en el conocimiento de la experiencia del paciente, nos permite realizar una fotografía de sus percepciones a nivel general, abordando los temas a nivel superficial.

Otra de las claves que nos proporciona el uso de esta técnica, es la utilización de escalas de medición, la facilidad de monitorizar los ítems a lo largo del tiempo, y la comparabilidad entre distintos contextos; por el contrario no es una buena técnica de cara al abordaje de temas sensibles para el paciente.

Por último, cabe mencionar las dificultades en cuanto a contar con participación de las personas en las encuestas, por lo que suele darse un alto índice de falta de respuesta suponiendo un sesgo importante en el uso de la técnica. Causa de esto puede ser debido a que es una técnica muy usada a nivel general desde distintos ámbitos de consumo y prestación de servicios, tanto en el ámbito público como privado, por lo que existe una "sobrecarga" en el consumidor/usuario. En contraposición, nos encontramos con una técnica que puede dar cobertura a una amplia población de referencia, siendo a la vez de fácil análisis de resultados, hecho que hace que su uso siga predominando en el campo sociosanitario.

Figura VII. Fortalezas y limitaciones de medir la experiencia del paciente a través de las encuestas $^{14}$

\begin{tabular}{|l|l|}
\hline \multicolumn{1}{|c|}{ Fortalezas } & \multicolumn{1}{|c|}{ Limitaciones } \\
\hline $\begin{array}{l}\text { Facilidad de utilizar escalas numéricas } \\
\text { para el análisis. }\end{array}$ & $\begin{array}{l}\text { Falta de capacidad para llegar a la } \\
\text { información en profundidad; tendencia } \\
\text { a la información superficial. }\end{array}$ \\
\hline $\begin{array}{l}\text { Existencia de herramientas validadas } \\
\text { que pueden ser utilizadas o adaptadas } \\
\text { para el contexto local. }\end{array}$ & $\begin{array}{l}\text { No cobertura de los aspectos más } \\
\text { delicados de la experiencia del } \\
\text { paciente como puede ser la dimensión } \\
\text { emocional. }\end{array}$ \\
\hline $\begin{array}{l}\text { Permite la estandarización (y por } \\
\text { tanto la comparación) de preguntas, } \\
\text { proceso y método de análisis. }\end{array}$ & $\begin{array}{l}\text { Laspersonas pueden sentir "sobrecarga } \\
\text { de la encuesta" ya que el método es } \\
\text { utilizado muy comúnmente (poco rigor } \\
\text { en la respuesta) }\end{array}$ \\
\hline $\begin{array}{l}\text { Permite el anonimato, el cúal permite } \\
\text { en coasiones que las personas se sientan } \\
\text { más cómodas al opinar. }\end{array}$ & $\begin{array}{l}\text { Puede excluir a personas que no } \\
\text { disponen de una buena alfabetización } \\
\text { y colectivos vulnerables. }\end{array}$ \\
\hline
\end{tabular}

14 De Silva, D. (2013) Measuring patient experience. Evidence Scan. The Health Foundation; 18. 
Calidad percibida y satisfacción: Cómo conocer y medir la experiencia del paciente...

\begin{tabular}{|l|l|}
\hline \multicolumn{1}{|c|}{ Fortalezas } & \multicolumn{1}{|c|}{ Limitaciones } \\
\hline $\begin{array}{l}\text { Facilidad de utilizar escalas numéricas } \\
\text { para el análisis. }\end{array}$ & $\begin{array}{l}\text { Falta de capacidad para Ilegar a la } \\
\text { información en profundidad; tendencia } \\
\text { a la información superficial. }\end{array}$ \\
\hline $\begin{array}{l}\text { Facilidad para participar gran número } \\
\text { de personas, de distribución (correo, en } \\
\text { línea etc.) y de análisis. }\end{array}$ & $\begin{array}{l}\text { Los resultados de las encuestas de } \\
\text { experiencia del paciente, no siempre } \\
\text { coinciden con otras fuentes de datos } \\
\text { sobre el servicio en cuanto a calidad } \\
\text { seguridad; estos datos no se pueden } \\
\text { utilizar solos como indicador de } \\
\text { calidad. }\end{array}$ \\
\hline $\begin{array}{l}\text { Permite realizar análisis de correlación } \\
\text { entre experiencia del paciente/ } \\
\text { satisfacción con resultados científico- } \\
\text { técnicos y clínicos. }\end{array}$ & $\begin{array}{l}\text { Las encuestas desarrolladas para un } \\
\text { contexto puede ser inadecuadamente } \\
\text { aplicado a otros. }\end{array}$ \\
\hline
\end{tabular}

Fuente: Elaboración propia a partir de De Silva, D. (2013) Measuring patient experience. Evidence Scan. The Health Foundation; $18^{15}$

\subsection{Grupo focal de pacientes}

La técnica de grupo focal tiene su origen en la sociología (Robert Merton ${ }^{16}$ ), extendiéndose posteriormente al ámbito de los estudios de mercado. En el ámbito de la salud, el uso de esta técnica es algo relativamente reciente, pero el aumento en su utilización viene siendo en los últimos años exponencial.

Como hemos visto en el capítulo dedicado a esta técnica, el sello característico de esta herramienta cualitativa (como entrevista grupal) es el uso explícito de la interacción para la producción de información sobre puntos de vista que sería menos posible acceder sin tal interacción del grupo. El grupo focal es «una conversación cuidadosamente planeada, diseñada para obtener información de un área definida de interés, en un ambiente permisivo y no directivo» ${ }^{17}$ cuyo propósito principal es obtener información cualitativa sobre un tema concreto para posteriormente analizarla de manera más detallada.

15 De Silva, D. (2013) Measuring patient experience. Evidence Scan. The Health Foundation; 18.

16 Merton RK (1987). The focused interview and focus groups: continuities and discontinuities. Public Opinion Quarterly.

17 Krueger RA. El grupo de discusión. Guía práctica para la investigación aplicada. Madrid: Pirámide, 1991. 
Concretamente, en el ámbito de la salud y calidad percibida, el grupo focal es una de las técnicas mejor indicadas cuando se desea profundizar en vivencias, experiencias y preferencias de los/as usuarios respecto a la atención prestada en un centro sanitario. A nivel metodológico, como veíamos en el tema anterior, el grupo focal es una herramienta de investigación/evaluación cualitativa, en la cual a través del diseño de una entrevista grupal semiestructurada (con preguntas estimulo en torno a aspectos concretos) se crea un espacio de opinión en el que hacer surgir vivencias y necesidades sentidas durante el propio proceso asistencial. Su uso es muy valioso, en cuanto que permite realizar un acercamiento a las experiencias de las personas atendidas y sus familias, además de la recopilación de conclusiones altamente relevantes para el desempeño diario en la atención al paciente.

En el marco del SESCAM, la aplicación de esta técnica se ha llevado a cabo en el marco del Comité de Calidad Percibida de la GAICR y también dentro del plan regional de humanización de la asistencia sanitaria puesto en marcha desde la Consejería de Sanidad (Plan Dignifica). Con el objeto de realizar un diagnóstico situacional de partida en algunos servicios y unidades concretas, se aplica esta herramienta en aras a obtener información a cerca de la opinión de los pacientes respecto a aspectos referidos a la humanización de la asistencia, aspectos que incorporamos en la entrevista como preguntas estímulo (Ver figura VIII).

Los resultados que te permite alcanzar un grupo focal son de gran validez subjetiva; como decimos surgen vivencias, sentimientos, experiencias... en definitiva resultados que no te permite obtener el uso de otras herramientas o técnicas. La interacción social entre personas consideradas entre ellas como semejantes por su vivencia común como pacientes de un servicio de salud, propicia una dinámica de expresión de opiniones e ideas subjetivas. No se trata de una técnica que tenga capacidad de representación o generación de resultados extrapolables a la población general, pero dispensa una profundidad en la información subjetiva que otras técnicas con mayor representatividad no alcanzan.

La realización de esta técnica muestra resultados especialmente sensibles y válidos para evidenciarnos áreas de mejora sobre las cuales trabajar y que repercutan directamente en mejorar la satisfacción. Además encontramos un valor añadido en el uso de esta herramienta: un potencial "humanizador" tanto para el paciente como el profesional. La herramienta brinda una oportunidad al paciente y a los profesionales que la desarrollan, de formar parte de un proceso participativo, de implicación en el cambio, y de alto componente emocional.

Por ello, puede y debe ser un fiel complemento a otras fuentes de información/evaluación sobre opinión y preferencias del paciente en nuestro sistema de salud, como pueden ser el análisis de reclamaciones o las encuestas de satisfacción; comparte las ventajas y desventajas de cualquier otro método cualitativo y algunas específicas. 
Frente a la entrevista individual, su principal ventaja es la interacción entre los participantes que como hemos dicho, estimula la generación de idea, pero puede convertirse en limitación si los participantes se sienten coartados por la presencia del grupo o por el dominio de la conversación de algunos miembros. Del mismo modo, es importante saber sortear los peligros potenciales de la técnica que provee la propia interacción: invertir tiempo en temas irrelevantes, perder el rumbo de los objetivos de investigación, disminuir la comparabilidad entre la información de diferentes grupos, etc ${ }^{18}$.

Como vemos, es una herramienta altamente válida en este ámbito, pero también decir que no es precisamente una opción fácil ya que "requiere cierto entrenamiento y una buena dosis de tolerancia para la incertidumbre". Del mismo modo, para una buena aplicación lleva tiempo diseñarla y organizarla así como para el análisis de los resultados, ya que conlleva un estudio más complejo y en profundidad de los datos. Por otro lado, dispone de una capacidad de empoderamiento y de motivación de las personas respecto a su compromiso con la mejora, que difícilmente favorecen otras técnicas.

Figura IX. Fortalezas y Limitaciones del uso de la técnica Grupo Focal de pacientes.

\begin{tabular}{|l|l|}
\hline \multicolumn{1}{|c|}{ Fortalezas } & \multicolumn{1}{|c|}{ Limitaciones } \\
\hline $\begin{array}{l}\text { Facilita la recopilación de información } \\
\text { en profundidad. }\end{array}$ & $\begin{array}{l}\text { No permite recopilar datos que se tra- } \\
\text { duzcan fácilmente en análisis numéricos } \\
\text { (No representatividad/inferencia) }\end{array}$ \\
\hline $\begin{array}{l}\text { Permite sondear las razones de las opi- } \\
\text { niones de la gente y profundizar en } \\
\text { pensamientos y sentimientos (Abordaje } \\
\text { temas delicados) }\end{array}$ & $\begin{array}{l}\text { Depende de la habilidad del entrevista- } \\
\text { dor o facilitador: puede pasar por alto } \\
\text { detalles específicos si no se realiza bien } \\
\text { o crear sesgos en la dinámica. }\end{array}$ \\
\hline $\begin{array}{l}\text { Ayuda a las personas a sentirse más } \\
\text { comprometidas en la mejora. }\end{array}$ & $\begin{array}{l}\text { Gran inversión de tiempo y disponibili- } \\
\text { dad por parte del paciente y/o familia }\end{array}$ \\
\hline $\begin{array}{l}\text { La utilización de un enfoque grupal, } \\
\text { favorece la retroalimentación de otros } \\
\text { miembros para la generación de ideas. }\end{array}$ & $\begin{array}{l}\text { Requiere esfuerzo en el análisis para } \\
\text { dar sentido a lo cualitativo detallado. }\end{array}$ \\
\hline
\end{tabular}

Fuente: Elaboración propia a partir de De Silva, D. (2013) Measuring patient experience. Evidence Scan. The Health Foundation; $18^{19}$.

18-16 MM García Calvente, I Mateo Rodríguez (2000) El grupo focal como técnica de investigación cualitativa en salud: diseño y puesta en práctica.

19 De Silva, D. (2013) Measuring patient experience. Evidence Scan. The Health Foundation; 18. 
Figura VIII. Grupo Focal en el Hospital General Universitario de Ciudad Real: Ejemplo. Preguntas estímulo.

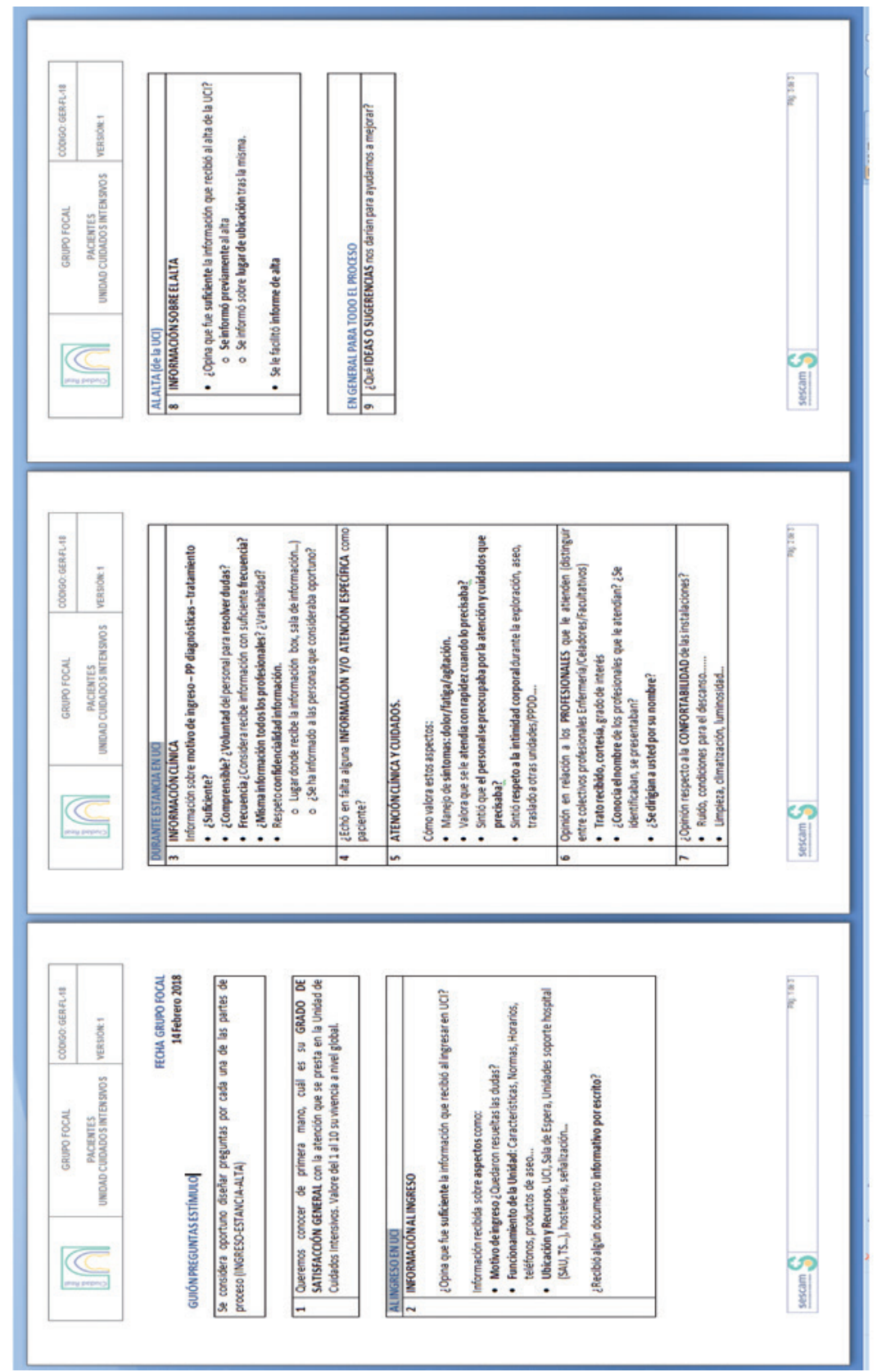




\section{A MODO DE CONCLUSIÓN...}

Mejorar la experiencia del paciente en un servicio de salud, debe ser objetivo clave en las organizaciones sanitaria; la década pasada ha traído un creciente interés en la comprensión de cómo las personas experimentan los servicios de salud. En una organización orientada al paciente y a la mejora de la atención que presta, se hace necesario el uso de herramientas que nos permitan aproximarnos a sus expectativas como usuarios, y detectar aquellos aspectos que sugieren mejorables, para poder así implementar mejoras en la gestión. La medición de la experiencia del paciente y la puesta en marcha de métodos y canales para escuchar su voz, "constituye una revolución silenciosa que paulatinamente va introduciendo un cambio en la cultura de las organizaciones" 20.

Como hemos visto, en el ámbito de la calidad percibida y medición de satisfacción del paciente, no existen fórmulas evidentes que nos indiquen qué herramienta utilizar de forma concreta; tampoco evidencias sobre la mejor y más efectiva en la indagación de experiencias. Por el contrario, cada una demuestra en su uso pros y contras, pero hay dos aspectos en los que principalmente podemos basar nuestra elección: nivel de profundidad de información al que queremos ahondar, y si queremos datos generalizables o no. Por otra parte, será necesario del adaptar el diseño a los contextos, tiempos disponibles y recursos tanto materiales como humanos con los que se cuenta. De todo modos, un único enfoque es probable que no cumpla todas las necesidades que nos planteamos, por lo que la combinación de las técnicas, puede ser siempre una buena opción buscando resultados complementarios.

En este sentido cabe destacar que hasta muy recientemente, la participación del paciente en el desarrollo de técnicas para medir la experiencia ha sido más bien superficial; tomando el camino de la corresponsabilidad en salud, debemos abrazar formas más colaborativas de participación de los pacientes, siendo las técnicas cualitativas las que disponen de un mayor potencial de empoderamiento y de implicación de paciente y profesionales en el cambio. Ésta es una de las áreas con un mayor potencial innovador en este sentido.

Esto combina con otra idea fundamental, en la cual cabe por último incidir: la medición de la satisfacción debemos entenderla como una herramienta en la gestión de los recursos y servicios sociosanitarios, pues nos sirve para tomar decisiones en base a lo que nos cuentan los pacientes respecto a la atención que perciben; es decir, debe servir para establecer estrategias en la gestión a corto, medio y largo plazo, en un ciclo de mejora continua. "La participación de los pacientes supone un nuevo modo de entender la relación entre éstos, los profesio-

20 Sociedad española de Atención al Usuario de la Sanidad. Claves para la Gestión de la Atención al Usuario en los Servicios de Salud. Barcelona: 2010. 
nales y los sistemas de salud, no solo desde el prisma del conocimiento y manejo de la propia salud a nivel individual, sino también desde la influencia que puede llegar a tener en la planificación de políticas sanitarias" ${ }^{21}$.

La literatura en este sentido, nos muestra que la medida en que está repercutiendo verdaderamente en resultados de mejora de la experiencia del paciente deja mucho que desear; el mero hecho de medir las experiencias del paciente no nos conduce necesariamente a mejoras en la atención ${ }^{22}$. La detección/medición de expectativas debe ser vista como una herramienta de ayuda para evaluar aspectos de la asistencia a lo largo del tiempo, y poder establecer indicadores que permitan un seguimiento en los cambios y tendencias. Es necesario propiciar la participación, pero con el fin de generar transformaciones concretas, no suponiendo una finalidad en sí misma, sigamos hacia la mejora.

21 Ruiz-Arazola A, Perestelo-Pérez I. Participación ciudadana en Salud: formación y toma de decisiones compartida. Informe SESPAS 2012. Gac Sanit.2012;26 (S):158-161.

22 Silva, D. Measuring patient experience. Evidence Scan. The Health Foundation. 2013; 18. 